\title{
INDIVIDUALIZED LUNG RECRUITMENT MANEUVER GUIDE BY PULSE-OXIMETRY IN ANESTHETIZED NON-OBESE PATIENTS UNDERGOING.
}

Villena A., , Jurado A., Carrizo J., Duca A., Soro M., Ferrando C.

Department of Anesthesiology and Intensive Care, Hospital Clínico Universitario de Valencia, Spain.

\section{BACKGROUND AND GOAL OF STUDY}

General anesthesia and mechanical ventilation promote atelectasis which negatively affects the lung function 1 and may promote postoperative pulmonary complications. The open-lung approach (OLA) is aimed at restoring the functional lung volume ${ }^{2}$. In a recent study, the $\mathrm{SpO}_{2}$ under low $\mathrm{FIO}_{2}$ was useful to monitor the lung's opening and closing pressure in morbidly obese anesthetized patients ${ }^{3}$.

Our goal was to test whether $\mathrm{SpO}_{2}$ can personalize the implementation of an open-lung approach during laparoscopy.

\section{MATERIALS AND METHODS}

$>30$ patients with SpO2 $\geq 97 \%$ on room-air before anesthesia were studied.

$>$ After anesthesia induction and capnoperitoneum the FlO2 was reduced to 0.21 .

$>$ Those patients whose Sp02 decreased below $97 \%$ complete the following phases: 1) First recruitment maneuver (RM), until reaching lung's opening pressure, defined as the inspiratory pressure level yielding a SpO2 $\geq 97 \%$; 2) a PEEP titration trial until reaching lung's closing pressure defined as the PEEP level yielding a SpO2 <97\%; 3) a second RM was performed and, 4) ongoing ventilation with PEEP adjusted above the detected closing pressure.

\section{RESULTS AND DISCUSSION}

Breathing air, 24 of 30 patients $(80 \%)$ showed SpO2 <97\% together PaO2/FIO2 <400mmHg.

The mean opening pressures were found at 40(6) and $33(4) \mathrm{cmH} 2 \mathrm{O}$ during the first and second RM, respectively.

The closing pressure was 11(5) cmH2O. This Sp02-guide approach reached an open-lung condition (table 1).

SpO2 discriminates the lung's opening and closing pressures with accuracy taking the reference PaO2/FIO2 (area under the receiver-) operating-curve of $0.88,95 \% \mathrm{Cl}: 0.80-0.97$ ).

\begin{tabular}{|c|c|c|c|c|c|}
\hline Variables & $\begin{array}{c}\text { Baseline } \\
\mathrm{SpO}_{2}< \\
97 \% \\
(\mathrm{n}=24)\end{array}$ & $\begin{array}{c}\text { Baseline } \\
\mathrm{SpO}_{2} \geq \\
97 \% \\
(\mathrm{n}=6)\end{array}$ & $\begin{array}{c}\text { p-value } \\
95 \% \mathrm{Cl} \\
\mathrm{SpO}_{2}<97 \% \text { vs } \\
\mathrm{SpO}_{2} \geq 97 \%\end{array}$ & $\begin{array}{c}\text { Open } \\
\text { Lung } \\
\text { Conditio } \\
n\end{array}$ & $\begin{array}{c}\text { p-value } \\
95 \% \mathrm{Cl} \\
\mathrm{SpO}_{2}<97 \% \text { vs } \\
\text { Open lung } \\
\text { Condition } \\
\end{array}$ \\
\hline $\mathrm{SpO}_{2}$ & $92(2)$ & $97(1)$ & $\begin{array}{c}<0.001 \\
3.1-7.2\end{array}$ & $98(1)$ & $\begin{array}{c}<0.001 \\
4.7-6.7\end{array}$ \\
\hline $\mathrm{PaO}_{2} / \mathrm{FlO}_{2}$ & $228(91)$ & $398(11)$ & $\begin{array}{c}<0.001 \\
92-247\end{array}$ & $436(76)$ & $\begin{array}{c}<0.001 \\
173-243\end{array}$ \\
\hline DPtp & $\begin{array}{l}18.0 \\
(7.5)\end{array}$ & $10.1(3.8)$ & $\begin{array}{c}0.02 \\
1.1-14.5\end{array}$ & $10.0(3.9)$ & $\begin{array}{c}<0,001 \\
5.3-10.4\end{array}$ \\
\hline Cdyn & $21(8)$ & $23(7)$ & $\begin{array}{c}0.57 \\
-9.6-5.4\end{array}$ & $35(16)$ & $\begin{array}{c}<0,001 \\
8.1-20.4\end{array}$ \\
\hline
\end{tabular}

\section{CONCLUSION}

\section{The non-invasive $\mathrm{SpO}_{2}$ value can easily individualize an open-lung approach.}

